\title{
A New Approach of Electronics and Photonics Convergence on Si CMOS Platform: How to Reduce Device Diversity of Photonics for Integration
}

\author{
Kazumi Wada \\ Department of Materials Engineering, The University of Tokyo, 7-3-1 Hongo, Bunkyo, Tokyo 113-8656, Japan \\ Correspondence should be addressed to Kazumi Wada, kwada@material.t.u-tokyo.ac.jp
}

Received 11 March 2008; Accepted 21 May 2008

Recommended by Pavel Cheben

Integrated photonics via Si CMOS technology has been a strategic area since electronics and photonics convergence should be the next platform for information technology. The platform is recently referred to as "Si photonics" that attracts much interest of researchers in industries as well as academia in the world. The main goal of Si Photonics is currently to reduce material diversity of photonic devices to pursuing CMOS-compatibility. In contrast, the present paper proposes another route of Si Photonics, reducing diversity of photonic devices. The proposed device unifying functionality of photonics is a microresonator with a pin diode structure that enables the Purcell effect and Franz-Keldysh effect to emit and to modulate light from SiGe alloys.

Copyright ( $) 2008$ Kazumi Wada. This is an open access article distributed under the Creative Commons Attribution License, which permits unrestricted use, distribution, and reproduction in any medium, provided the original work is properly cited.

\section{INTRODUCTION}

Electronic and photonic integrated circuits (EPICs) on $\mathrm{Si}$ complementary metal oxide semiconductor (CMOS) platform have widely been studied to achieve higher figure-ofmerits in communications and computations. A challenging issue is that there are so many kinds of devices in photonics as in Table 1. The photonic devices have been developed for optical communication systems in which integration of these devices on a chip has never been important. Thus, the concurrent photonics consist of various devices (materials) such as light emitters (III-V semiconductors), modulators $(\mathrm{LiNbO} 3)$, interconnects $\left(\mathrm{SiO}_{2}\right)$, filters $\left(\mathrm{SiO}_{2}\right)$, photodetectors (III-V semiconductors), and isolators (YIG). On the contrary, electronics as in Table 1 consists only transistor and interconnect; and the material systems are all compatible to the CMOS fabrication process referred to as "CMOScompatible." Si photonics today have focused on reduction of the material diversity by fabricating these photonic devices with CMOS compatible materials. In the present paper we will propose a new approach: functional unification of photonic devices. The enabler of the unification is the materials system of $\mathrm{Si}$ waveguide and $\mathrm{SiO}_{2}$. The features of the system are as follows: (i) polarity and conductivity control of Si waveguides by implementing pin diode structures,

(ii) high-index contrast to $\mathrm{SiO}_{2}$ acting as cladding and electrical insulation that reduces device footprints.

To utilize these features, the proposed novel device is based on a microresonator with a built-in pin diode. Physics presenting emitter and modulator functions are the Purcell effect [1] and Franz-Keldysh effect [2].

\section{HOW TO REDUCE DEVICE DIVERSITY}

The Purcell effect is known to enhance spontaneous emission rate on a resonance wavelength of a microresonator, as schematically shown in Figure 1(a). There have been various papers reporting the effect is actually working based on standing wave resonators $[3,4]$ and traveling wave resonators $[5,6]$. On the other hand, electro-optic effect such as the plasma effect or Franz-Keldysh effect is known to change the complex refractive indices of the resonator materials, which functions to modulate optical amplitudes by shifting the resonance wavelength as also schematically in Figure 1(b). Here, we would propose a new device to unify several photonic functions in Table 1. The device is a resonator with 
TABLE 1: Device and material diversity of electronics and photonics.

\begin{tabular}{llc}
\hline & Devices & Materials \\
\hline \multirow{2}{*}{ Electronics } & Transistor & $\mathrm{Si}, \mathrm{SiO}_{2}$ \\
& Interconnect & $\mathrm{Al}, \mathrm{Cu}$ \\
\hline \multirow{7}{*}{ Photonics } & Light emitter & $\mathrm{III}-\mathrm{V}$ material \\
& Modulator & $\mathrm{LiNbO}_{3}$ \\
& Interconnect & $\mathrm{SiO}_{2}$ \\
& Filter & $\mathrm{SiO}_{2}$ \\
& Detector & $\mathrm{III}-\mathrm{V}$ material \\
& Amplifier & $\mathrm{III}-\mathrm{V}$ material \\
& Isolator & YIG \\
& $\cdots$ & $\cdots$ \\
\hline
\end{tabular}

a pin structure as in Figure 1(c). Forward-biasing of the resonator would function as a light emitter due to the Purcell effect; and reverse-biasing would function as a modulator due to the Franz-Keldysh effect. The device is referred to as a unified functional device (UFD) in the present paper. UFDs should in principle function as a light emitter, an amplifier, a modulator, a switch, as well as a tunable filter. Accordingly the device diversity of photonics in Table 1 is to dramatically shrink and the device set for photonic integration is only UFDs, detectors, and interconnects. Isolator function is not yet available.

\section{PHYSICS AND THE REQUIREMENTS TO UNIFY THE DEVICE FUNCTIONS}

In this section we will discuss physics, materials, and structures of UFDs.

\subsection{Purcell effect and the requirements to ring resonators}

We first consider a single localized radiating dipole; its spontaneous emission rate $R$ can be expressed via the Fermi golden rule;

$$
R=\frac{1}{\tau}=\frac{4 \pi^{2}}{h}\left|\left\langle\Psi_{f}\left|H_{i}\right| \Psi_{i}\right\rangle\right|^{2} \cdot \rho
$$

where $h$ is the Planck constant, $\rho$ is the density of optical modes (states) at the emitter's angular frequency. Considering the dipole moment $\mathbf{u}$, and the electrical vector $\mathbf{E}$, the term $\left|\left\langle\Psi_{f}\left|H_{i}\right| \Psi_{i}\right\rangle\right|^{2}$ can be simplified as $\left|E\left\langle\Psi_{f}|u| \Psi_{i}\right\rangle\right|^{2}$. The matrix element depends on the band structure of the media, we just refer to it as $u^{2}$ for simplification later. When $\mathrm{E}$ is parallel to $\mathbf{u}$, the spontaneous emission rate can be expressed in the following well-known form:

$$
R(r)=\int_{0}^{\infty} \frac{4 \pi^{2}}{h} u^{2}(r, \omega) E^{2}(r, \omega) \cdot f_{c}\left(1-f_{v}\right) \rho(\omega) d \omega .
$$

Here, $f_{c}$ and $f_{v}$ are the electron density of the conduction band and valence band expressed by Fermi-Dirac statistics.

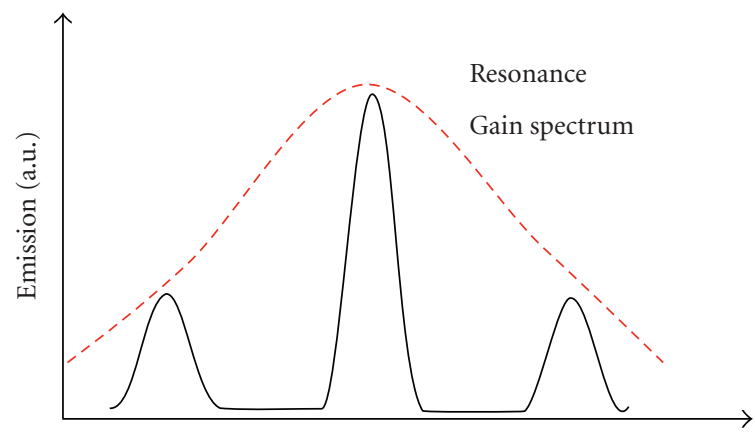

(a)

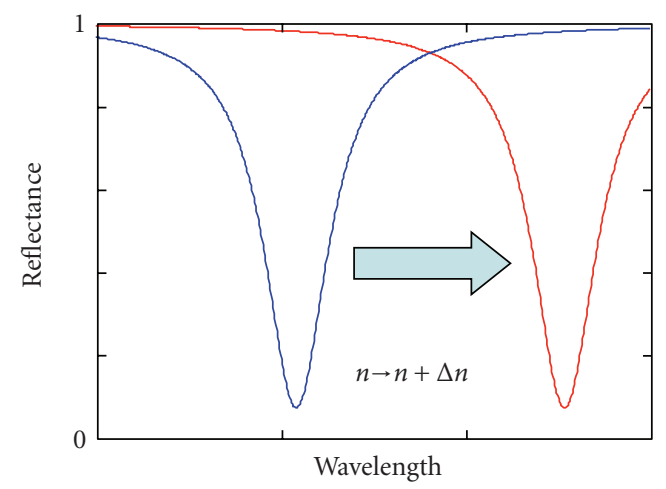

(b)

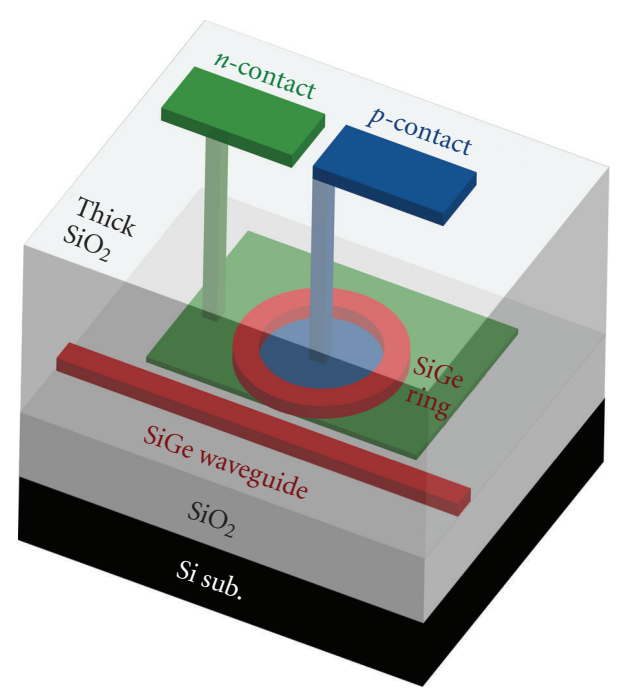

(c)

FIGURE 1: Schematic diagrams of physics of unified function devices and a possible structure.

$\rho(\omega) d \omega$ denotes the mode density in a unit of angular frequency. Equation (2) indicates that $R$ depends on the initial electron-and hole density of states and the final photonic density of states. The quantum confinement focuses on increasing $\mathbf{u}$ while the microresonator on controling $\mathbf{E}^{2}$.

When the modal volume of the resonator is very large, the optical mode is continuous in the $k$-space. Assuming 
the distribution of the electron is uniform all around the structure, $R$ can be expressed as

$$
R_{\mathrm{sp}} \cong \frac{u_{0}^{2} \omega_{\mathrm{sp}}^{2} n_{\mathrm{eff}}}{3 \pi \varepsilon_{0} c^{3}}
$$

where $\omega_{\text {sp }}$ denotes the center of the spontaneous emission band, $u_{0}$ the average dipole momentum, $n_{\text {eff }}$ the effective refractive index of the resonator. Considering a small resonator with the scale of the emission wavelength $\lambda_{s}$, the modes will be quantized in the $k$-space. Meanwhile, the electrical field $\mathbf{E}$ will increase with resonator size reduction. The emission rate at the resonance wavelength can be derived as

$$
R_{\mathrm{sp}} \cong \frac{4 u_{0}^{2} \omega_{c} \Gamma_{r}\left(\omega_{c}\right)}{3 \varepsilon_{0} n_{\mathrm{eff}}^{2} V_{c} \Delta \omega_{i}},
$$

where $\Gamma_{r}\left(\omega_{c}\right)$ is the relative confinement factor, expressing the overlapping between the optical mode and the resonator structure, and can be 1 in the high-index contrast systems. Here, $V_{c}$ is the modal volume, and $\Delta \omega_{i}$ is the larger one of $\Delta \omega_{\text {sp }}$ or the full width at half maximum (FWHM) of resonance mode $\left(\Delta \omega_{c}\right)$. Since spontaneous emissions are generally broader than the resonance modes, then $\Delta \omega_{i}=$ $\Delta \omega_{c} ; \Delta \omega_{c} / \omega_{c}=Q$. Here, we assumed the resonance frequency is at the center of the spontaneous emission band.

Finally, the enhancement of the spontaneous emission rate usually called Purcell factor $F_{p}$ can be expressed by comparing the emission rates in the small resonator and space:

$$
F_{p}=\frac{p \Gamma_{r}}{4 \pi^{2}} \cdot\left(\frac{\lambda}{n_{\mathrm{eff}}}\right)^{3} \cdot \frac{Q}{V_{c}} .
$$

In silicon resonators, the other factors expect $Q$ and $V_{c}$ are all state constant. The higher $\mathrm{Q} / V_{c}$, the higher Purcell factor. According to Zsai et al., we need to get an enhancement factor of nearly 100 to get positive net gain when the loss is due to free-carrier absorption [7]. So, our requirement of the Purcell enhancement is at least 100.

\subsection{Franz-Keldysh effect and requirements to unify device functions}

$\mathrm{Si}$ and Ge have the inversion symmetry in the lattice, prohibiting the existence of a linear electro-optic effect. Thus, the bias applications to the pin diode structures have been employed to change in carrier concentration and/or electric field strength and eventually altering refractive indices of the structures. The Franz-Keldysh (FK) effect is a typical one having been used in III-V semiconductor-based modulators. However, the effect was ignored until recently in $\mathrm{Si}$, since the effect in Si was known very weak. We have recently reported that Ge shows a large electro-optic coefficient induced by the FK effect. The difference from Si can be explained by the difference in the band structure of $\mathrm{Ge}$ where the $\Gamma$ point causing the FK effect is only slightly above the $L$ valley.

The FK effect occurs as follows: the change in dielectric constant $(\Delta \varepsilon)$ at energy $E$ under the field $F$ is given in terms of the well-known FK expressions $G(\eta)$ and $F(\eta)$ containing Airy functions and their derivatives $[8,9]$ :

$$
\begin{gathered}
\Delta \varepsilon(E, F)=\left(B / E^{2}\right)(\hbar \theta)^{1 / 2}[G(\eta)+i F(\eta)], \\
\hbar \theta=\left(\frac{e^{2} \hbar^{2} F^{2}}{2 \mu}\right)^{1 / 3}, \quad \eta=\frac{E_{g}-E}{\hbar \theta} .
\end{gathered}
$$

Here, $B$ represents transition probability by absorption of photon, containing matrix element $E_{p}$ and reduced effective mass $\mu$. $\hbar$ is Planck's constant $h$ divided by $2 \pi$. In our model, we considered the FK effect from the direct band edge as noted above. The contribution from the indirect band edge was ignored in this treatment.

According to a simple mathematics, the following relations can be derived:

$$
Q=\frac{\lambda}{\Delta \lambda}=\frac{n}{\Delta n} .
$$

Here, $\Delta \lambda$ denotes the shift of resonance wavelength $\lambda$, and $\Delta n$ denotes the shift of refractive index $n$. Assuming that $Q$ of the resonator in Figure 1 is 4000 , and that $\Delta n$ should be $10^{-3}$ when $n$ is 4.0 , that is, Ge, the modulation depth can be $50 \mathrm{~dB}$ or higher, which is more than enough. However, we will find out that $\Delta n=10^{-3}$ is the realistic limit in terms of FK effect as shown later. So, we set our goal of index change to be mid $10^{-4}$. Thus, $Q$ of $10^{4}$ must be required.

\subsection{Ring as a microresonator}

There are two kinds of microresonators utilizing traveling waves and standing waves. We have employed traveling wave resonator to demonstrate the feasibility of UFDs since it is expandable to a three-terminal device. In the traveling wave resonator, we have further chosen ring resonators instead of disks because of fewer mode numbers involved in the resonator. In general, the observed $Q$ can be written by [10]

$$
\frac{1}{Q_{\text {total }}}=\frac{1}{Q_{0}}+\frac{1}{Q_{\text {ext }}} \text {. }
$$

Here, $Q_{\text {total }}$ is the observed $Q, Q_{0}$ loss $Q$ of the ring itself, and $Q_{\text {ext }}$ coupling $Q$ between the waveguide and ring. In case of isolated ring resonators, $Q_{\text {total }}=Q_{0}$. Thus, $Q_{\text {total }}$ can be expressed as

$$
Q_{\text {total }}=Q_{0}=\frac{\omega_{0}}{2 \alpha v_{g}} .
$$

Here, $\alpha$ denotes the overall attenuation coefficient of the ring, usually consisting of material absorption and attenuation due to light scattering of the ring waveguide.

These are simple explanations to control device functions and requirements for UFDs to function.

\section{RESULTS}

In this section, we will present the simulation and experimental demonstration of the characteristics of ring resonators functioning as light emitters and modulators. We 


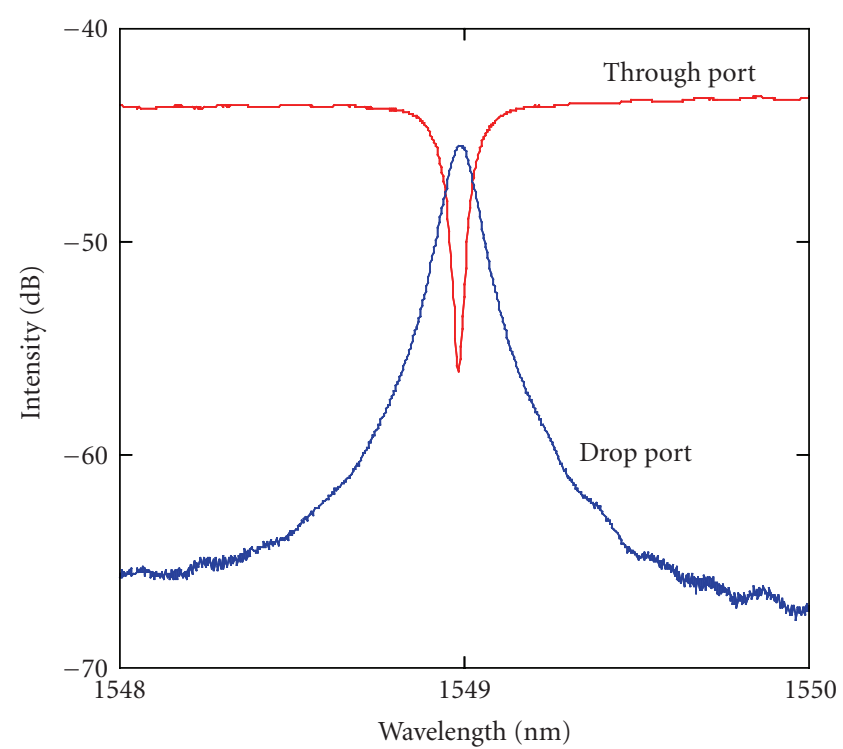

(a)

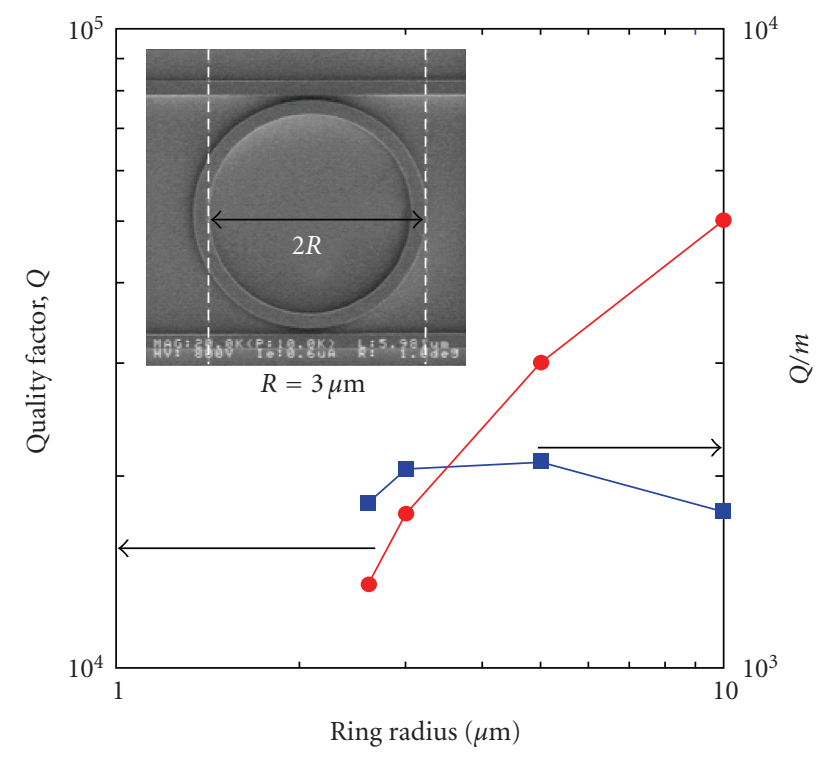

(b)

FIgURE 2: Transmission spectra of the ring resonator and $Q$ and $Q / m$ versus ring radii. $Q$ is quality factor and $m$ is the number of wavelengths in the longitudinal ring mode.

have not yet prototyped those with pin diode structure. The resonators were fabricated on $\mathrm{Si}$ on insulator (SOI) wafers by means of electron beam (EB) lithography and dry etching (DE) $[6,8,11]$. The SOI wafer has a $3 \mu \mathrm{m}$ buried oxide (BOX) layer and a $200 \mathrm{~nm}$ top Si layer. The waveguides used to verify the feasibility of UFDs have $400 \mathrm{~nm}$ width and $200 \mathrm{~nm}$ height where the transverse mode is single. The radius ranged from 2.6 to $10 \mu \mathrm{m}$. The gap between incoming waveguide and the ring was changed from $150 \mathrm{~nm}$ to $350 \mathrm{~nm}$. The gap between outgoing waveguide and the ring is identical to the one between the incoming waveguide and the ring. The SEM image of the fabricated sample is shown as insert in Figure 2. The waveguides for emission function have $250 \mathrm{~nm}$ width and $370 \mathrm{~nm}$ height. The excitation is done by $\mathrm{Ar}^{+}$ion laser irradiation focusing on the waveguide. The resonator is isolated, and light escaping from the ring is collected using optical microscope.

\subsection{Required characteristics of Si ring resonator and the experimentally obtained data}

Figure 2 shows $Q$ and $Q / m$ of the various sizes of rings to check if the requirements described in Section 2 would be met. $Q$ has to be larger than $10^{4}$ for FK requirement for modulator. The cavity volume $V$ is $m\left(\lambda / n_{\text {eff }}\right)^{3}$, thus the term of $\left(\left(\lambda / n_{\text {eff }}\right)\right)^{3} Q / V_{c}$ in (5) can be simplified by $Q / m$, where $m$ denotes the number of the wavelength in the longitudinal mode of interest. To meet the requirement of 10-100 time Purcell enhancement, $Q / m>10^{3}$. Figure 2(a) shows transmission spectra of the through port and drop port of the ring resonator, $r=10 \mu \mathrm{m}$ and gap $=300 \mathrm{~nm}$. From the transmission spectrum of the drop port, $Q$ is determined to be nearly 50000 . It is also clear from Figure 2(b) that the

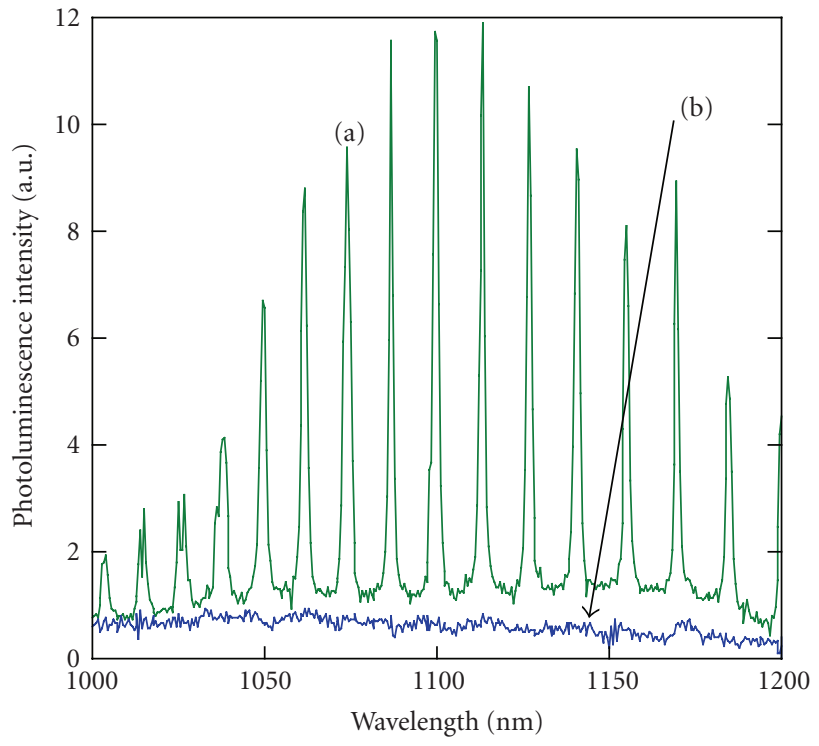

Figure 3: Photoluminescence spectra of (a) ring and (b) surrounding slab. The ring is $3.2 \mu \mathrm{m}$ in radius. The peaks are generated at the resonance wavelengths.

rings fabricated have met the Purcell requirement as well as the FK modulation when the radius is $2.6-10 \mu \mathrm{m}$.

\subsection{Purcell enhancement of spontaneous emission of the ring resonator}

Figure 3 shows the photoluminescence spectra of (a) the isolated Si ring resonator with the radius of $3.2 \mu \mathrm{m}$ and the width of $250 \mathrm{~nm}$ and of (b) the slab region surrounding the ring. The luminescence of the slab in Figure 3 is multiplied 


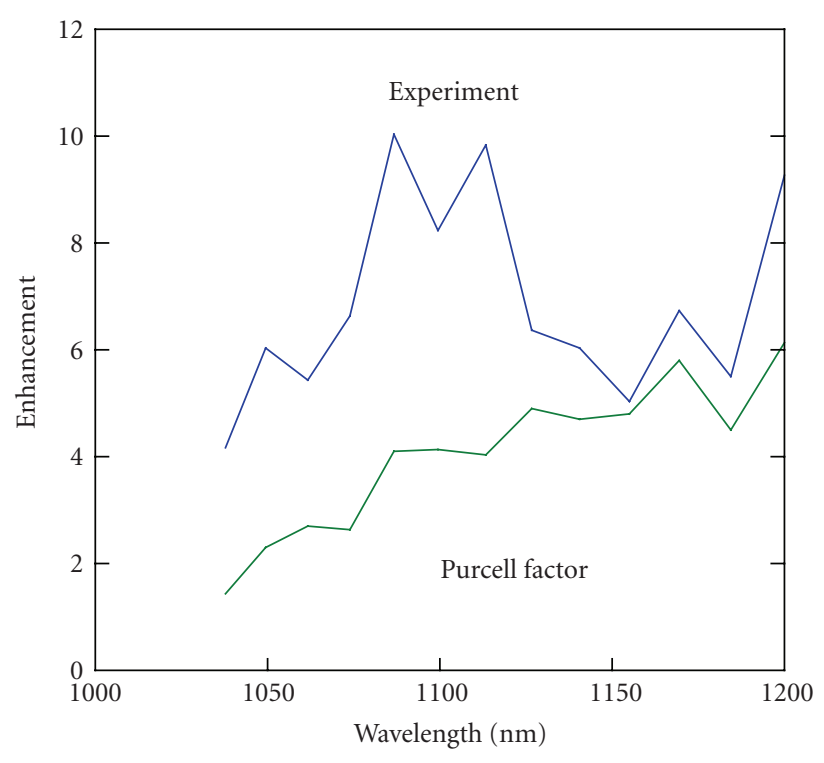

FIgURE 4: Comparison between the experimental enhancements and theoretical enhancement (Purcell factor).

by 10 to show it in this linear scale range. The luminescence consists of many peaks and the highest peak is more than 100-times stronger in intensity than the luminescence of the slab. The wavelengths of all peaks show a clear one-to-one correspondence with the resonance frequencies of the ring. Figure 4 shows the luminescence enhancement observed and the Purcell factors calculated by (5). The experimental data are in a good agreement with the prediction, indicating that the enhancement would occur due to the Purcell Effect. The enhancement is 10 times which is lower than our requirement of ring resonator. We will discuss this point later on.

\subsection{Resonance shift using FK effect}

The Ge $\mathrm{p}-\mathrm{i}-\mathrm{n}$ photodiodes used in this work were fabricated from $1.3 \mu \mathrm{m}$ thick undoped Ge epilayers on a $\mathrm{p}^{+} \mathrm{Si}(100)$ substrate with boron concentration $>10^{19} \mathrm{~cm}^{-3}$. The Ge epilayer was under $0.20 \%$ tensile strain [12]. Phosphorus was implanted into a poly-Si layer that was deposited on the top of the Ge epilayer to create a pin diode. External biases of 0 and $5 \mathrm{~V}$ were applied to the diode, corresponding to electric fields of 14 and $70 \mathrm{kV} / \mathrm{cm}$ in the Ge i-layer. The absorption coefficient $\alpha$ was calculated from the photodiode responsivity $(R)$ defined by photocurrent normalized by incoming light power in A/W. Figure 5 shows the absorption coefficients extracted from the spectral responsivity. As predicted by the FK effect, the absorption coefficient increases with the applied electric field.

We have incorporated the strain effect into our FranzKeldysh model to analyze the data as in Figure 5. The excellent fitting of the experimental data to the model obtained, suggesting that the change in absorption coefficients is due to the Franz-Keldyshi effect in the strained Ge epilayer on Si. Based on the Kramers-Kronig relation, we have derived

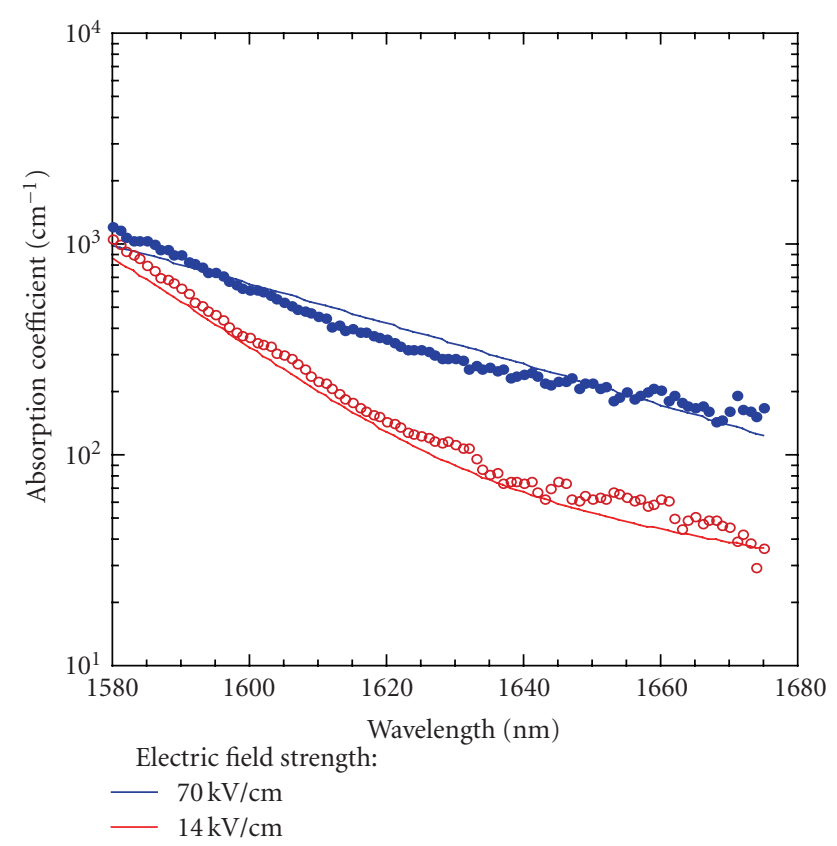

FIGURE 5: Absorption coefficient spectra under electric-field applications. The experiments are well reproduced by the Franz-Keldysh analysis.

the dependences of refractive indices difference $\Delta n$ and absorption coefficient $\alpha$ as a function of wavelength as in Figure 6. Figure 6(a) shows $\Delta n$ as a parameter of electric field strength, 20,50 , and $100 \mathrm{kV} / \mathrm{cm}$. It is found that the electric field strength $20 \mathrm{kV} / \mathrm{cm}$ is not enough to change refractive index, mid $10^{-4}$ to enable modulator function. The Ge pin diode for under electric field strength $50 \mathrm{kV} / \mathrm{cm}$ can realize such index change in the wavelength range of $1750 \mathrm{~nm}$ or shorter. However, the absorption coefficient gets larger in the wavelength range as in Figure 6(b) and it is less likely that $Q$ should exceed $10^{4}$ according to (9). Indeed, Figure 6(c) indicates that $Q$ should be mid $10^{3}$ at $1700 \mathrm{~nm}$ that is lower than the requirement discussed above. As in Figure 6(c), $Q$ of the Ge ring under $100 \mathrm{kV} / \mathrm{cm}$ can reach $\sim 10^{4}$ at $1850 \mathrm{~nm}$. This lead us to conclude that the Ge-based ring can be operated as a modulator at $1850 \mathrm{~nm}$ under the electric field, $100 \mathrm{kV} / \mathrm{cm}$.

\section{DISCUSSION}

We have shown the microring resonators can unite the photonic functions ranging from emitter, modulator, as well as filter. This suggests it can function as amplifier, and switch. This substantially reduces device diversity in Photonics. Since materials used in the UFDs are $\mathrm{Si}$ or Ge, that is, CMOS-compatible, the presented approach reducing device diversity should be beneficial for large-scale integration of electronics and photonics on a Si chip. However, the presented UFDs require two material systems $\mathrm{Si}$ and $\mathrm{Ge}$ for resonators although these are on the same materials platform. In this section, we will consider the feasibility of 


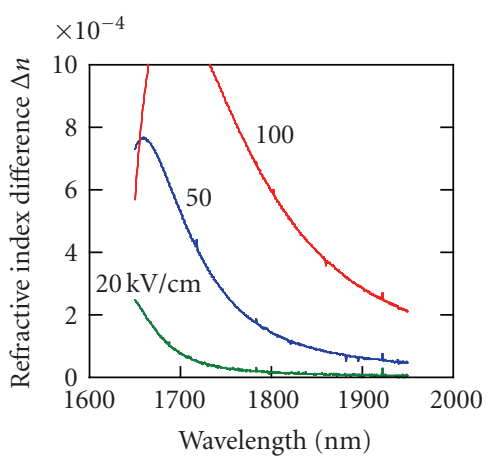

(a)

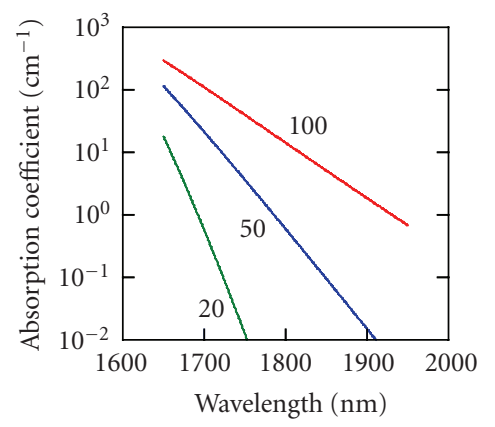

(b)

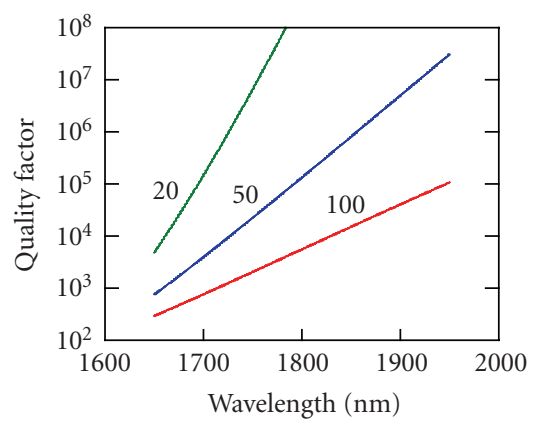

(c)

Figure 6: Changes in (a) refractive index and (b) absorption coefficient derived by Kramers-Kronig relation. The theoretical dependence of (c) $Q$ versus wavelength.

materials unification via $\mathrm{SiGe}$ as the resonator materials to unify the emitter and modulator functions.

\subsection{SiGe UFDs}

Because of low absorption coefficient resulting in high $Q$, both of the Purcell enhancement and the FK shift are large at the photon energy slightly below gap, like $1200 \mathrm{~nm}$ for Si. According to the literature, Ge-rich SiGe, for example, the Ge composition $\sim 0.95$ should be of such materials. The indirect bandgap is around $0.8 \mathrm{eV}$, the communication wavelength band called C- and L-band, and the direct bandgap referencing the FK effect is $\sim 1.0 \mathrm{eV}$ [9]. The tensile strain of $\mathrm{Ge}$ on $\mathrm{Si}$ reduces the direct gap to $0.9 \mathrm{eV}$, as in Figure 7. The reduction of direct bandgap observed was in a good agreement with the deformation potential calculations [13]. Because of tensile-trained SiGe, we would be able to design UFDs built on the unique material system. This will be reported in a separate paper.

\subsection{Increase of luminescence enhancement}

The Si ring resonator emits strong light at the resonance wavelengths at the indirect band edge, $1.1 \mu \mathrm{m}$. The enhancement we obtained was about 10 as in Figure 4 . To get higher enhancement, we need to increase $Q$. Although $Q$ of the isolated ring is not ready for measurement, the Purcell analysis leads us to estimate that $Q$ is as low as $\sim 500$, corresponding the overall attenuation coefficient to be $\sim 180 \mathrm{~cm}^{-1}$ according to (9), assuming the group velocity is $1 \times 10^{10} \mathrm{~cm} / \mathrm{s}$ and wavelength $1.1 \mu \mathrm{m}$. It is clear that the attenuation cannot be material absorption, $1.3 \mathrm{~cm}^{-1}$ at $1.1 \mu \mathrm{m}$. It has been pointed that the sidewall roughness should increase the attenuation coefficient and could explain $180 \mathrm{~cm}^{-1}$. According to Soref and Bennett [14], free-electron absorption is $\sim 1 \mathrm{~cm}^{-1}$ at the density of $10^{18} / \mathrm{cm}^{3}$ at $1.1 \mu \mathrm{m}$ and free holes do almost the same. When the ring resonator is excited to measure photoluminescence, a high density of carriers if it is in concentration of $10^{20} \mathrm{~cm}^{-3}$ would result in such loss in terms of in free-carrier absorption. $Q_{0}$ should be reduced unless otherwise. Therefore, passivation of $\mathrm{Si}$

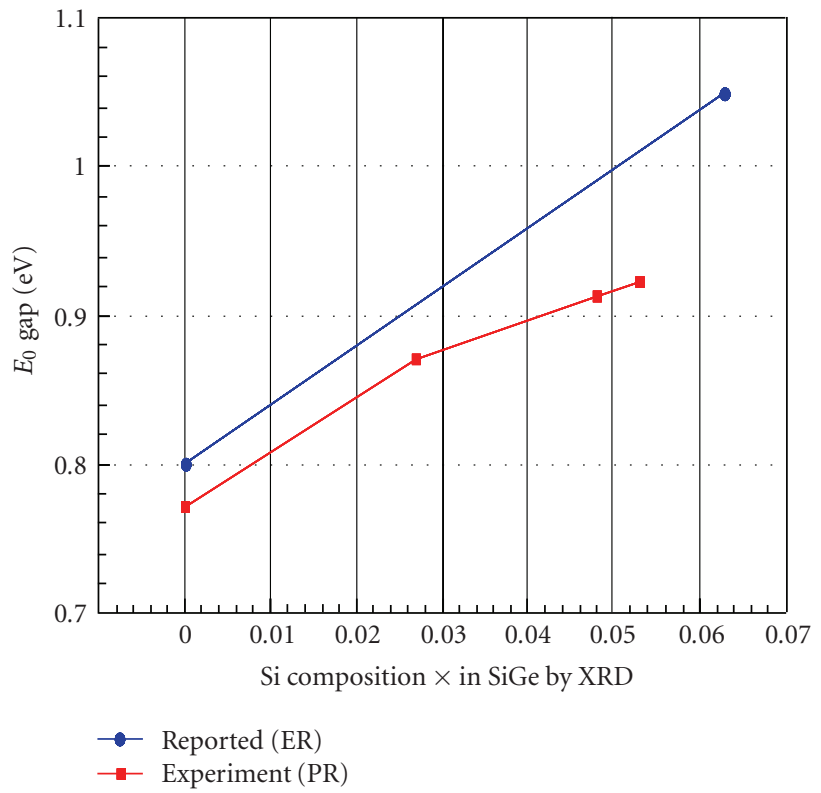

Figure 7: The direct bandgap versus SiGe alloy composition. The reported data were taken from bulk Ge using electroreflectance. Our data based on photoreflectance shows smaller direct bandgap in terms of tensile-strain in Ge epi on Si.

waveguides such as hydrogenation, and oxidation should be indispensable for UFDs.

\section{CONCLUSION}

A new approach on electronics and photonics convergence on Si CMOS platform is proposed; reducing device diversity rather than material diversity in photonics. The device concept unifying functional devices is a microresonator with a pin diode structure, and is theoretically and experimentally studied. It has been shown that the Purcell effect and the Franz-Keldysh effect should enable devicing the concept to unite photonic functions such as emitter, amplifier, modulator, switch, and filter. It is further discussed that Ge-rich SiGe would be the material system for UFDs. 
The present approach simplifies photonic devices to UFDs, photodetectors, and interconnects, that will be beneficial for large-scale integrated electronics and photonics circuits.

\section{ACKNOWLEDGMENTS}

The authors acknowledge Dr. Christina Manolatou in Cornel University for the FDTD simulator code. The work was partly supported by Grant-in-Aid for Creative Scientific Research by Japan Society for the Promotion of Science. The devices were fabricated at the NTT Microsystem Integration Laboratories, by the University of Tokyo VLSI Design and Education Center's (VDEC) 8-inch EB writer F5112+VD01 donated by ADVANTEST Corporation with the collaboration of Cadence Corporation, and at Massachusetts Institute of Technology. The authors are grateful to Drs. K. Yamada, T. Tsuchizawa, T. Watanabe, and S. Itabashi at NTT Microsystem Integration Laboratories, and to Drs. J. F. Liu, S. Jongthammanurak, J. Michel, and L. C. Kimerling at Massachusetts Institute of Technology for their in-depth discussions. The authors also like to thank Dr. Y. Ishikawa, Mr. S. Y. Lin, and Mr. P. H. Lim.

\section{REFERENCES}

[1] E. M. Purcell, "Spontaneous emission probabilities at radio frequencies," Physical Review, vol. 69, no. 11-12, p. 681, 1946.

[2] M. Cardona, Modulation Spectroscopy, Academic Press, New York, NY, USA, 1969.

[3] M. Zelsmann, E. Picard, T. Charvolin, et al., "Seventy-fold enhancement of light extraction from a defectless photonic crystal made on silicon-on-insulator," Applied Physics Letters, vol. 83, no. 13, pp. 2542-2544, 2003.

[4] T. Baba, D. Sano, K. Nozaki, K. Inoshita, Y. Kuroki, and F. Koyama, "Observation of fast spontaneous emission decay in GaInAsP photonic crystal point defect nanocavity at room temperature," Applied Physics Letters, vol. 85, no. 18, pp. 39893991, 2004.

[5] J. S. Xia, Y. Ikegami, K. Nemoto, and Y. Shiraki, "Observation of whispering-gallery modes in Si microdisks at room temperature," Applied Physics Letters, vol. 90, no. 14, Article ID 141102, 3 pages, 2007.

[6] S. Lin, Y. Kobayashi, Y. Ishikawa, and K. Wada, "Luminescence enhancement by Si ring resonator structures on silicon on insulator," Applied Physics Letters, vol. 92, no. 2, Article ID 021113, 3 pages, 2008.

[7] C.-Y. Tsai, "Theoretical model for the optical gain coefficient of indirect-band-gap semiconductors," Journal of Applied Physics, vol. 99, no. 5, Article ID 053506, 5 pages, 2006.

[8] Y. Miki, K. Yamada, T. Watanabe, et al., "Design of spontaneous emission enhancement based on Si ring resonators," in Proceedings of the Electrochemical Society SiGe Symposium, Pennington, NJ, USA, 2005.

[9] J. S. Kline, F. H. Pollak, and M. Cardona, "Electroreflectance in the Ge-Si alloys," Helvetica Physica Acta, vol. 41, pp. 968-976, 1968.

[10] H. A. Haus, Waves and Fields in Optoelectronics, Prentice-Hall, Englewood Cliffs, NJ, USA, 1984.

[11] T. Tsuchizawa, K. Yamada, H. Fukuda, et al., "Microphotonics devices based on silicon microfabrication technology," IEEE Journal on Selected Topics in Quantum Electronics, vol. 11, no. 1, pp. 232-239, 2005.
[12] Y. Ishikawa, K. Wada, D. D. Cannon, J. Liu, H.-C. Luan, and L. C. Kimerling, "Strain-induced band gap shrinkage in Ge grown on Si substrate," Applied Physics Letters, vol. 82, no. 13, pp. 2044-2046, 2003.

[13] D. D. Cannon, J. Liu, D. T. Danielson, et al., "Germanium-rich silicon-germanium films epitaxially grown by ultrahigh vacuum chemical-vapor deposition directly on silicon substrates," Applied Physics Letters, vol. 91, no. 25, Article ID 252111, 3 pages, 2007.

[14] R. A. Soref and B. R. Bennett, "Electrooptical effects in silicon," IEEE Journal of Quantum Electronics, vol. 23, no. 1, pp. 123129, 1987. 

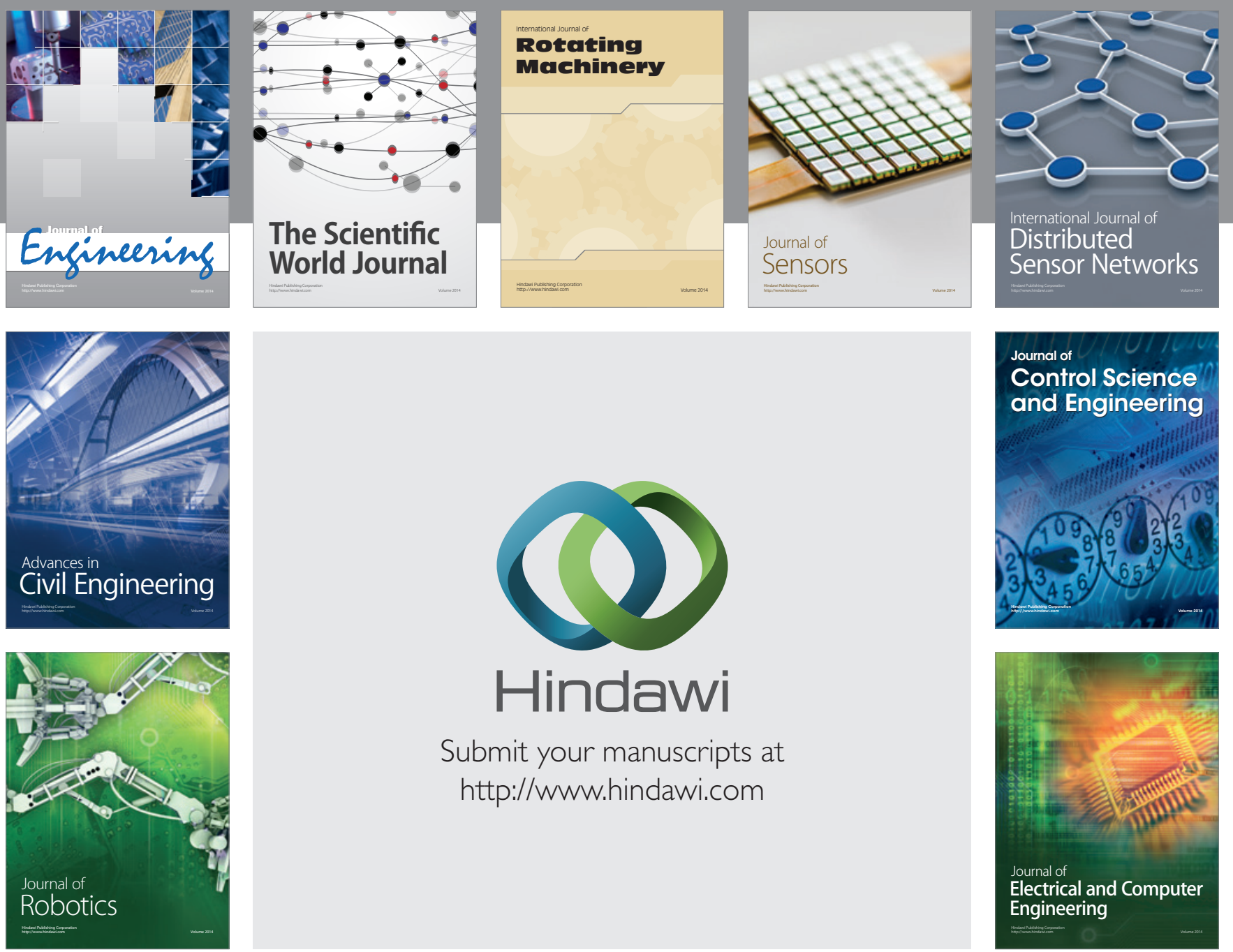

Submit your manuscripts at

http://www.hindawi.com
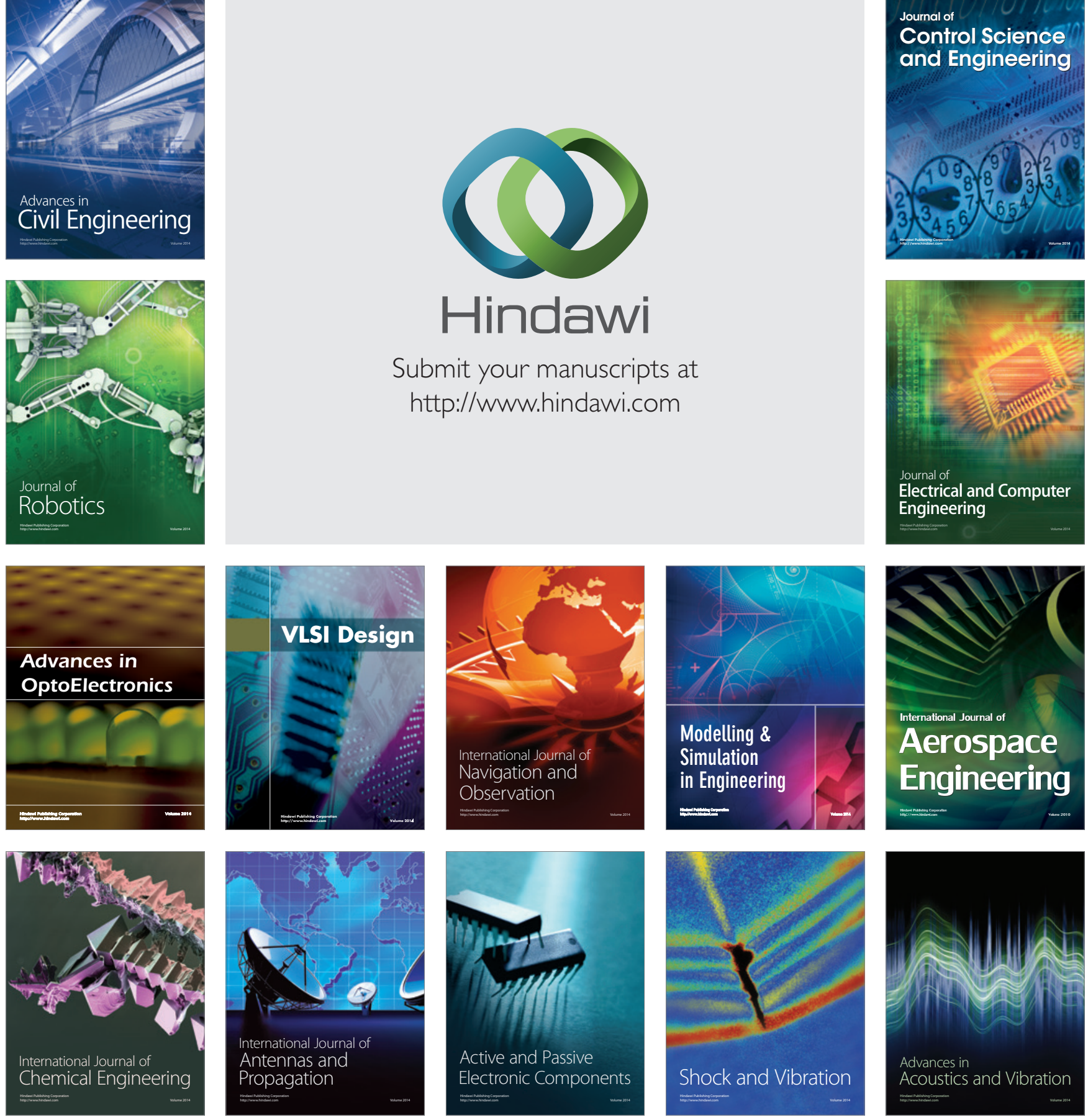